

\section{ONTARIO MATHEMATICAL MEETINGS}

The fourth Ontario Mathematical Meeting was held Saturday, March 25, 1967 at Sidney Smith Building, University of Toronto. Research papers were presented in the morning, followed by lunch at Sir Daniel Wilson Residence, followed by a one-hour address by Professor Irving Kaplansky (University of Chicago) entitled: Quadratic Forms. Abstracts of research papers which were presented are as follows:

67.7 J. Brenner and L. Beasley (University of British Columbia) Bounds for Certain Permanents and Determinants.

If a matrix has dominant main diagonal, a bound can be given for the permanent of the matrix in terms of the absolute values of its elements. The result is similar to that of Price (Proc. Amer. Math. Soc. 2 (1951), 497 502), but the proof is different; relations among the permanents of the various minors of the matrix must be used (see Duke J., 1959). For a matrix block diagonally dominant, (definition given in Proc. Nat. Acad. Sci. 40 (1954), 452-454), bounds for the determinant and in some cases also for the permanent can be given.

67.8 D. Klarner (McMaster University)

The Number of Non-Isomorphic K-Coloured Graphs.

A $k$-coloured graph of type $\left(n_{1}, \ldots, n_{k}\right)$ is a set of coloured nodes $\mathrm{N}_{\mathrm{n}_{1}}^{1} \cup \ldots \cup \mathrm{N}_{\mathrm{n}_{\mathrm{k}}}^{\mathrm{k}}$, where $N_{r}^{s}=\{(s, j): j=1, \ldots, r\}$, and an edge set $E$ contained in the complete edge set $\left.\left\{\{c, j),\left(c^{\prime}, j^{\prime}\right)\right\}:(c, j) \varepsilon N_{n_{c^{\prime}}}^{c^{\prime}},\left(c^{\prime}, j^{\prime}\right) \varepsilon N_{n_{c^{\prime}}}^{c^{\prime}}, 1 \leq c<c^{\prime} \leq k\right\} ;$ the complete $k$-coloured graph $G\left(n_{1}, \ldots, n_{k}\right)$ of type $\left(\mathrm{n}_{1}, \ldots, \mathrm{n}_{\mathrm{k}}\right)$ has the complete edge set as its edge set. 
Let $S_{n}$ denote the symmetric group on $N_{n}=\{1, \ldots, n\}$. Two k-coloured graphs $X$ and $Y$ of type $\left(n_{1}, \ldots, n_{k}\right)$ are isomorphic if there is a $\pi \varepsilon S_{k}$ such that $n_{i}=n_{\pi i}, i=1, \ldots, k$, and if there are $\varphi_{1} \varepsilon S_{n_{1}}, \ldots, \varphi_{k} \varepsilon S_{n_{k}}$ such that

(1) $\left(\pi: \varphi_{1}, \ldots, \varphi_{k}\right)^{*}:\left\{(c, j),\left(c^{\prime}, j^{\prime}\right)\right\} \rightarrow\left\{\left(\pi c, \varphi_{c} j\right),\left(\pi c^{\prime}, \varphi_{c} j^{\prime}\right)\right\}$ transforms the edge set of $\mathrm{X}$ into that of $\mathrm{Y}$.

The cycle index of a permutation $\theta$ of degree $n$ written as the product of disjoint cycles is $Z(\theta: \bar{x})=x_{1}{ }_{1} x_{2}{ }_{2}^{i}, \ldots$, where $\theta$ has exactly $i_{t}$ cycles of length $t$. The cycle index of a permutation group $G$ of degree $n$ is $|G|^{-1} Z(G: \bar{x})=|G|^{-1} \sum Z(\theta: \bar{x}), \theta \varepsilon G$.

Let $\Gamma^{*} \mathrm{G}\left(\mathrm{n}_{1}, \ldots, \mathrm{n}_{\mathrm{k}}\right)$ denote the permutation group of edge automorphisms (as defined as in (1)) of the complete $k$-coloured graph of type $\left(n_{1}, \ldots, n_{k}\right)$. According to Pólya's theorem, the number of non-isomorphic k-coloured graphs of type $\left(n_{1}, \ldots, n_{k}\right)$ with exactly e edges is the coefficient of $x^{e}$ in the polynomial

$$
\left|\Gamma^{*} \mathrm{G}\left(\mathrm{n}_{1}, \ldots, \mathrm{n}_{\mathrm{k}}\right)\right|^{-1} \mathrm{Z}\left(\Gamma^{*} \mathrm{G}\left(\mathrm{n}_{1}, \ldots, \mathrm{n}_{\mathrm{k}}\right): \mathrm{x}+1, \mathrm{x}^{2}+1, \ldots\right) \text {. }
$$

Our result is an algorithm for computing the cycle index of $\Gamma^{*} \mathrm{G}\left(\mathrm{n}_{1}, \ldots, \mathrm{n}_{k}\right)$ in terms of the cycle indices of certain symmetric groups. We have calculated these cycle indices for all tricoloured graphs of type $\left(n_{1}, n_{2}, n_{3}\right)$, where $\left(n_{1}, n_{2}, n_{3}\right)$ is a partition of $n, 3 \leq n \leq 9$.

(Harary, On the Number of Bi-Coloured Graphs, Pacific J. of Math. 8 (1958), 743-755, described an algorithm for computing the relevant cycle indices for two coloured graphs). 
Let $R$ be a commutative ring with unity, $\mathbb{C}(R)$ the category of all left $R$-modules. Let $\Gamma$ be a positively fully ordered semigroup with zero which is sup-complete. Definition: - A generalised length function on $R$ is a "mapping" L: $C(R) \rightarrow \Gamma U\{\infty\}$ s.t

$$
L(\overline{0})=0 \quad \overline{0} \in C(R)
$$

(2) $0 \rightarrow E^{\prime} \rightarrow E \rightarrow E^{\prime \prime} \rightarrow 0$ exact $\Longrightarrow L(E)=L\left(E^{\prime}\right)+L\left(E^{\prime \prime}\right)$

(3) $L(E)=\sup \{L(X) \mid X \subseteq E, X$ a finitely generated module $\}$.

This generalises the concept introduced by D.G. Northcott and M. Reufel (A generalisation of the concept of length, Quart. Jour. of Math. Oxford, Vol.16 (1965), 297-321). We determine all the generalised length functions on a valuation ring $R$ with valuation $v$, and extend some of their main theorems.

Sample theorems.

THEOREM 1. Let $R$ be an integral domain, $L$ a length function on $R$ to $\Gamma$ with $0<L(R)<\infty$. Then for every $E \in C(R), L(E)=$ Rank $E . L(R)$.

THEOREM 2. Let $(R, v, \Gamma)$ be a valuation ring, $L$ a length function on $\mathrm{R}$ to $\bar{\Gamma}$ such that

$\left\{\alpha \mid \alpha \in \mathrm{R}, \mathrm{L}\left(\frac{\mathrm{R}}{\alpha \mathrm{R}}\right)=\infty\right\}=(0)$ and $\left\{\alpha \mid \alpha \in \mathrm{R}, \mathrm{L}\left(\frac{\mathrm{R}}{\alpha \mathrm{R}}\right)>0\right\}$

is the maximal ideal of $R$. Then there exists a map $\varphi: \Gamma^{+} \rightarrow \bar{\Gamma} \operatorname{s.t}(\varphi \circ v)(\alpha)=L\left(\frac{R}{\alpha R}\right)$ and $\varphi \circ v$ is an equivalent valuation to $\mathrm{V}$. Further, for every ideal $\mathrm{A}$ in $\mathrm{R}$, $L\left(\frac{R}{A}\right)=\inf _{\alpha \in A} L\left(\frac{R}{\alpha R}\right)$.

THEOREM 3. Let $(R, v, \Gamma)$ be a valuation ring where $\Gamma$ is a totally ordered group. Let $\left(\Gamma^{+}\right)^{*}=\bar{\Gamma}$ be the completion of the positive part of $\Gamma$. Then there exists a length function on $R$ to $\bar{\Gamma}$ such that for every R-ideal A , $L\left(\frac{R}{A}\right)=\inf _{\alpha \in A} v(\alpha)$. 
We prove that if $k$ is odd and $0\left(x^{2 / 3}\right)$, then

$$
\begin{aligned}
\underset{\substack{1<\mathrm{n}<\mathrm{X} \\
\mathrm{n} \equiv \mathrm{b}(\bmod \mathrm{k})}}{ } \mathrm{r}(\mathrm{n}) & =\frac{\pi}{4} \mathrm{H}_{\mathrm{k}} \overline{(\mathrm{B}) \frac{\mathrm{X}}{\mathrm{k}}}+0\left(\mathrm{x}^{2 / 3} \mathrm{k}^{-1 / 2} \mathrm{~d}_{3}(\mathrm{k})\right) \\
& +0\left(\mathrm{x}^{(2 / 3)+\beta_{\mathrm{k}}-(1 / 2)(1+3 \beta)}(\mathrm{b}, \mathrm{k})^{1 / 2} \mathrm{~d}_{3}(\mathrm{k})\right)
\end{aligned}
$$

for $0<\beta<1 / 2$, where

$$
\mathrm{H}_{k}(b)=\sum_{q / k} \frac{X(q)}{q} C_{q}(b),
$$

$\mathrm{X}(\mathrm{q})$ is the non-principal character modulo $4, \mathrm{C}_{\mathrm{q}}(\mathrm{b})$ is the Ramanujan sum and $d_{3}(k)$ is the number of representations of $k$ as a product of three factors. A similar result holds for $k$ even. A direct consequence of this is that for $(b, k)=1$, there exists a solution to

$$
x^{2}+y^{2} \equiv b(\bmod k)
$$

such that $x, y=0\left(k^{3 / 4}\right)$, provided, $k$ has a bounded number of factors.

\subsection{Rudolf Wille (McMaster University) \\ Affine Coordinatization of Abstract Geometries.}

An abstract geometry is an order pair (,$C$ ) consisting of a set $S$ and an algebraic closure operator $C$ on the power set of $S$ under which the empty set and singletons are closed (B. Jónsson, Lattice-theoretic approach to projective and affine geometry, Symposium on the Axiomatic Method (1959), 188-203). (S, C) is said to be affine coordinatizable if and only if the lattice of all subspaces (i.e. all $\mathrm{X} \subseteq \mathrm{S}$ with $\mathrm{C}(\mathrm{X})=\mathrm{X}$ ) is isomorphic to the lattice of all congruence classes of an universal algebra, which is therefore called an affine coordinatization algebra.

THEOREM 1. Every universal algebra is an affine coordinatization algebra.

For characterization of the affine coordinatizable geo- 
metries we generalize the concepts which are used to coordinatize affine geometries (E. Artin, Geometric algebra, 1957). A weak parallelism on an abstract geometry $(\mathrm{S}, \mathrm{C})$ is a reflexive binary relation on the set of all subspaces, the domain of which is the set of all lines (i.e. all $\mathrm{C}(\mathrm{p}, \mathrm{q})$ with $\mathrm{p} \neq \mathrm{q}$ in $\mathrm{S})$, furthermore:

(i) If $\mathrm{L} \Pi \mathrm{P}, \mathrm{P} \supseteq \mathrm{M}$ and $\mathrm{M} \Pi \mathrm{Q}$, then there is an $\mathrm{R} \supseteq \mathrm{Q}$ with $L \Pi R$.

(ii) If $L \Pi P$ and $p \in P$, then $P \subseteq C(p, L)$.

(iii) For $L$ and $p$ there is at most one $P$ with $L \Pi P$ and $p \in P$.

A $\Pi$-dilatation is a mapping $\delta$ from $S$ into itself such that $\mathrm{p} \delta \neq \mathrm{q} \delta$ implies there is a $\mathrm{P}$ with $\mathrm{C}(\mathrm{p}, \mathrm{q}) \Pi \mathrm{P}$ and $\mathrm{p} \delta, \mathrm{q} \delta \in \mathrm{P}$.

THEOREM 2. An abstract geometry (S, C) is affine coordinatizable if and only if $(S, C)$ satisfies the following two conditions:

(i) $\mathrm{C}(\mathrm{p}, \mathrm{q}, \mathrm{r}) \subseteq \mathrm{X}$ whenever $\mathrm{p}, \mathrm{q}, \mathrm{r} \in \mathrm{X}$ implies $\mathrm{C}(\mathrm{X})=\mathrm{X}$.

(ii) There exists a weak parallelism $\Pi$ such that $\mathrm{p} \varepsilon \mathrm{C}(\mathrm{q}, \mathrm{r}, \mathrm{s})$ implies thereare $\pi$-dilatations $\delta_{1}, \ldots, \delta_{\mathrm{n}}$ with $q \varepsilon\{q, r, s\} \delta_{1},\{q, r, s\} \delta_{i} \cap\{q, r, s\} \delta_{i+1} \neq \phi$ for $\mathrm{i}=1, \ldots, \mathrm{n}-1$ and $\mathrm{p} \varepsilon\{\mathrm{q}, \mathrm{r}, \mathrm{s}\} \delta_{\mathrm{n}}$.

THEOREM 3. An affine geometry $(S, C)$ is affine coordinatizable if and only if the dilatation group generated by all dilatations $\delta$ with $p \delta=q$ is transitive on $C(p, q)$.

THEOREM 4. A projective geometry $(S, C)$ is affine coordinatizable if and only if $S$ is a line or every line consists of exactly two points.

Theorem 4 states that the lattice of all congruence classes of a non-simple universal algebra is modular if and only if it is distributive.

67.12 S. Golab (University of Waterloo) On Inner Product of Two Vectors.

Suppose that in a vector space the norm $\Omega(x)$ of the vector $x$ fulfills only the axioms:

(1) $\Omega(\mathrm{x}) \geq 0$,

(2) $\Omega(x)=0 \Longleftrightarrow x=\theta$ ( $\theta$ denotes the zero vector $)$ 
(3) $\Omega(\lambda \mathrm{x})=\lambda \Omega(\mathrm{x})$ for $\lambda \geq 0$

We set

$$
\varphi(\mathrm{x}, \mathrm{y})=\frac{1}{2}\left\{\Omega^{2}(\mathrm{x})+\Omega^{2}(\mathrm{y})-\Omega^{2}(\mathrm{y}-\mathrm{x})\right\}, \Phi(\mathrm{x})=\Omega^{2}(\mathrm{x}) .
$$

The question: 'when does $\varphi(x+y, z)=\varphi(x, z)+\varphi(y, z)$ ?' leads to the functional equation: $\Phi(x+y)+\Phi(z)-\Phi(z-x-y)=$ $\Phi(x)+\Phi(z)-\Phi(z-x)+\Phi(y)+\Phi(z)-\Phi(z-y)$. We show that because of the non-negativity of $\Phi$, all solutions of the above equation determine the euclidean metric in our vector space (we use a result of J. Aczél; the theorem of Jordan and von Neumann cannot be applied here).

\subsection{F.V. Atkinson (University of Toronto)}

Sums of powers of complex numbers.

The problem of determining

$$
\begin{aligned}
& M_{n}=\min \left\{\max _{1 \leq k \leq n}\left|\sum_{r=1}^{n} z_{r}^{k}\right|\right\} \text { for all } z_{1}, \ldots, z_{n} \\
& \text { with } \max \left|z_{r}\right|=1,
\end{aligned}
$$

was raised by $P$. Turán who conjectured that $M_{n} \rightarrow 1$ as $n \rightarrow \infty$.

I showed (On sums of powers of complex numbers, Acta. Math. Acad. Sci. Hung. 12 (1961), 185-188) that $M_{n}>1 / 6$, and subsequently (On sums of powers of complex numbers; an improved estimate, Math. Research Center, Technical Summary Report \#428, Madison, December 1963) that $M_{n}>1 / 3$. In the present investigation the method of these papers is pushed to its natural limit, to give the Theorem. For large $n, M_{n}$ exceeds the root, lying between $1 / 3$ and $\pi / 8$, of the equation

$$
\frac{s^{2}}{\pi} \int_{0}^{\infty}\left|\exp 2 s \int_{0}^{\varphi / 2}\right| \frac{\sin x}{x}|d x-1|^{2}\left|\int_{\varphi}^{\infty} \frac{e^{-i u}}{u} d u\right|^{2} d \varphi=1 \text {. }
$$


67.14 K. Murasugi (University of Toronto)

Interchangeability of Links.

A link $\ell$ consisting of two knots $K$ and $L$ in 3-sphere $\mathrm{S}^{3}$ is said to be interchangeable if there exists an autohomeomorphism of $S^{3}$ that maps $K$ onto $L$ and $L$ onto $\mathrm{K}$. If $l$ is interchangeable then $K$ and $L$ must be of the same type. This is obviously a necessary but insufficient condition.

THEOREM. Suppose that a link $\ell=K \cup L$ is interchangeable. Assume that $\mathrm{K}$ is a trivial knot and that the linking number of $K$ and $L$ is not zero. Then necessarily the Alexander polynomial of $l$ is symmetric, i.e. $\Delta(\mathrm{x}, \mathrm{y})=\Delta(\mathrm{y}, \mathrm{x})$.

67.15 T.M. Viswanathan (Queen's University) o-Tensor Products.

Let $A$ be an ordered ring and $\mathrm{M}_{\mathrm{A}^{\prime} \mathrm{A}} \mathrm{N}$ ordered modules over the ordered ring A. We construct an ordered abelian group $\mathrm{M}_{\mathrm{A}} \bigotimes_{\mathrm{A}}^{\mathrm{O}} \mathrm{N}$, called the o-tensor product of the ordered modules $M$ and $N$, as the solution of a universal problem. An example is given to show that

$\circ$

$\mathrm{M} \otimes \mathrm{N}$ may not coincide (as an abelian group) with A

$\mathrm{M} \otimes \mathrm{N}$. We determine a set of sufficient conditions for A

0

$M \otimes N$ and $M \otimes N$ to coincide and deduce thereby that the o-tensor product of two semi-closed abelian groups coincides with the usual tensor product. We prove that

0

$M \otimes N$ satisfies properties analogous to those of A

$\mathrm{M} \otimes \mathrm{N}$ - for example $\mathrm{B}^{\mathrm{M}} \mathrm{A} \otimes \mathrm{A}^{\mathrm{N}}$ is an ordered A A

B-module in a natural way, when $B$ is a directed ring. 
